\title{
An Investigation of Uranyl Sulfate Complexation under Hydrothermal Conditions by Quantitative Raman Spectroscopy and Density Functional Theory
}

Christopher D. Alcorn, Jenny S. Cox, Lucas M. S. G. A. Applegarth, and Peter R. Tremaine*

'Department of Chemistry, University of Guelph, Guelph, ON, Canada, N1G 2W1

*E-mail address: tremaine@ uoguelph.ca

Supplementary Information 
Table S.1 Integrated Raman peak areas of uranyl and sulfate species versus temperature for Solution $1\left[0.702 \mathrm{~mol} \cdot \mathrm{kg}^{-1} \mathrm{UO}_{2} \mathrm{SO}_{4}+\right.$ $0.375 \mathrm{~mol} \cdot \mathrm{kg}^{-1} \mathrm{H}_{2} \mathrm{SO}_{4}$ ], in arbitrary units

\begin{tabular}{ccccccccccc}
\hline$T /{ }^{\circ} \mathrm{C}$ & $\mathrm{UO}_{2}{ }^{2+}$ & St. Error & $\mathrm{UO}_{2} \mathrm{SO}_{4}{ }^{0}$ & St. Error & $\mathrm{UO}_{2}\left(\mathrm{SO}_{4}\right)_{2}{ }^{2-}$ & St. Error & $\mathrm{SO}_{4}{ }^{2-}$ & St. Error & $\mathrm{HSO}_{4}{ }^{-}$ & $\mathrm{St}_{\text {. Error }}$ \\
\hline 25 & 5139.0 & 1291.1 & 15536.8 & 2792.3 & 21603.3 & 1443.0 & 1582.5 & 1243.2 & 3800.8 & 2827.0 \\
75 & 2061.7 & 257.4 & 10230.2 & 208.2 & 30431.9 & 233.7 & 2030.3 & 19892.2 & 3672.9 & 11545.3 \\
125 & 1706.1 & 443.5 & 10057.6 & 244.1 & 27752.8 & 521.9 & 1134.1 & 4057.9 & 3302.4 & 4411.6 \\
175 & 1742.1 & 460.2 & 11016.1 & 213.8 & 25897.8 & 997.2 & 1611.8 & 15023.5 & 3639.8 & 3283.1 \\
225 & 1236.6 & 354.3 & 11532.5 & 1248.7 & 21377.1 & 3479.1 & 874.7 & 481.7 & 2321.7 & 1018.1 \\
275 & 1219.1 & 533.7 & 15005.6 & 2523.8 & 14411.4 & 3236.5 & 1916.3 & 7601.0 & 4827.5 & 858.8 \\
325 & 346.7 & 271.3 & 14759.2 & 1017.5 & 13605.0 & 823.9 & 985.7 & 292.4 & 3640.0 & 481.8 \\
\hline
\end{tabular}

Table S.2 Integrated Raman peak areas of uranyl and sulfate species versus temperature for Solution $2\left[0.514 \mathrm{~mol}^{\circ} \mathrm{kg}^{-1} \mathrm{UO}_{2} \mathrm{SO}_{4}+\right.$ $0.275 \mathrm{~mol} \cdot \mathrm{kg}^{-1} \mathrm{H}_{2} \mathrm{SO}_{4}$ ], in arbitrary units

\begin{tabular}{ccccccccccc}
\hline$T /{ }^{\circ} \mathrm{C}$ & $\mathrm{UO}_{2}{ }^{2+}$ & St. Error & $\mathrm{UO}_{2} \mathrm{SO}_{4}{ }^{0}$ & St. Error & $\mathrm{UO}_{2}\left(\mathrm{SO}_{4}\right)_{2}{ }^{2-}$ & St. Error & $\mathrm{SO}_{4}{ }^{2-}$ & St. Error & $\mathrm{HSO}_{4}{ }^{-}$ & $\mathrm{St}^{-}$Error \\
\hline 25 & 2461.4 & 1591.2 & 8658.6 & 3413.0 & 15549.3 & 1767.3 & 1307.3 & 1509.0 & 3068.9 & 961.0 \\
75 & 915.3 & 281.5 & 6686.4 & 260.4 & 20685.7 & 242.7 & 1700.8 & 7051.8 & 2411.3 & 7061.6 \\
125 & 584.9 & 170.5 & 6221.8 & 213.4 & 18601.6 & 244.1 & 1270.2 & 12126.4 & 2986.4 & 6040.8 \\
175 & 906.9 & 343.7 & 5631.9 & 172.4 & 14038.4 & 779.8 & 793.8 & 9628.1 & 2423.9 & 3374.4 \\
225 & 788.9 & 123.2 & 7019.7 & 103.7 & 12423.5 & 1711.3 & 1162.5 & 3493.0 & 2715.0 & 2535.1 \\
275 & 946.6 & 131.7 & 7173.5 & 141.5 & 7770.7 & 704.2 & 874.4 & 2662.5 & 1796.5 & 958.7 \\
325 & 0.0 & 27.2 & 13259.0 & 944.7 & 3991.1 & 899.3 & 835.9 & 1002.5 & 2427.9 & 617.4 \\
\hline
\end{tabular}


Table S.3 Integrated Raman peak areas of uranyl and sulfate species versus temperature for Solution $3\left[0.317 \mathrm{~mol} \cdot \mathrm{kg}^{-1} \mathrm{UO}_{2} \mathrm{SO}_{4}+\right.$ $0.169 \mathrm{~mol} \cdot \mathrm{kg}^{-1} \mathrm{H}_{2} \mathrm{SO}_{4}$ ], in arbitrary units

\begin{tabular}{ccccccccccc}
\hline$T /{ }^{\circ} \mathrm{C}$ & $\mathrm{UO}_{2}{ }^{2+}$ & St. Error & $\mathrm{UO}_{2} \mathrm{SO}_{4}{ }^{0}$ & St. Error & $\mathrm{UO}_{2}\left(\mathrm{SO}_{4}\right)_{2}{ }^{2-}$ & St. Error & $\mathrm{SO}_{4}{ }^{2-}$ & St. Error & $\mathrm{HSO}_{4}{ }^{-}$ & $\mathrm{St}^{2}$ Error \\
\hline 25 & 3242.1 & 837.2 & 9412.7 & 2020.0 & 14112.4 & 885.8 & 1863.2 & 1873.9 & 3190.6 & 3002.7 \\
75 & 801.9 & 338.5 & 6830.3 & 354.2 & 20254.1 & 291.0 & 2052.3 & 6595.1 & 1945.4 & 9580.8 \\
125 & 1005.4 & 537.0 & 6712.3 & 271.3 & 19155.8 & 751.5 & 1152.3 & 17598.5 & 2569.4 & 5750.5 \\
175 & 974.8 & 549.7 & 6301.4 & 275.3 & 16394.0 & 1265.7 & 832.8 & 14341.4 & 3080.2 & 4229.6 \\
225 & 772.6 & 154.3 & 7019.9 & 143.1 & 11795.5 & 1842.7 & 1002.4 & 4304.3 & 2204.0 & 3036.4 \\
275 & 249.3 & 195.6 & 3695.7 & 680.5 & 5004.0 & 630.8 & 188.3 & 1745.2 & 1301.6 & 993.1 \\
325 & 0.0 & 429.9 & 6409.1 & 1312.6 & 6592.4 & 1282.7 & 427.3 & 306.0 & 1452.8 & 457.5 \\
\hline
\end{tabular}

Table S.4 Integrated Raman peak areas of uranyl and sulfate species versus temperature for Solution $4\left[0.757 \mathrm{~mol} \cdot \mathrm{kg}^{-1} \mathrm{UO}_{2} \mathrm{SO}_{4}+\right.$ $0.842 \mathrm{~mol} \cdot \mathrm{kg}^{-1} \mathrm{H}_{2} \mathrm{SO}_{4}$ ], in arbitrary units

\begin{tabular}{|c|c|c|c|c|c|c|c|c|c|c|}
\hline$T /{ }^{\circ} \mathrm{C}$ & $\mathrm{UO}_{2}{ }^{2+}$ & St. Error & $\mathrm{UO}_{2} \mathrm{SO}_{4}{ }^{0}$ & St. Error & $\mathrm{UO}_{2}\left(\mathrm{SO}_{4}\right)_{2}{ }^{2-}$ & St. Error & $\mathrm{SO}_{4}{ }^{2-}$ & St. Error & $\mathrm{HSO}_{4}^{-}$ & St. Error \\
\hline 25 & 3285.2 & 96.8 & 10765.1 & 79.1 & 29279.2 & 259.1 & 1693.5 & 1610.4 & 6643.7 & 2045.0 \\
\hline 75 & 2143.6 & 175.4 & 9781.9 & 139.7 & 31044.4 & 519.3 & 3522.0 & 16515.1 & 7878.8 & 9044.1 \\
\hline 125 & 2220.7 & 375.7 & 10506.2 & 116.0 & 28683.7 & 415.5 & 1881.0 & 2595.4 & 7289.5 & 4862.9 \\
\hline 175 & 1965.4 & 358.4 & 12038.0 & 177.9 & 23553.1 & 725.2 & 1564.1 & 11139.3 & 7916.1 & 3407.8 \\
\hline 225 & 1521.5 & 351.4 & 15869.5 & 1146.1 & 17540.3 & 2567.3 & 3494.1 & 1902.0 & 5332.2 & 3153.0 \\
\hline 275 & 231.8 & 131.9 & 21386.5 & 669.6 & 13786.1 & 913.0 & 2896.7 & 1313.8 & 5877.3 & 2637.1 \\
\hline 325 & 65.8 & 167.5 & 18286.4 & 4422.4 & 15948.9 & 4317.5 & 1127.8 & 3487.1 & 6392.5 & 1427.8 \\
\hline 350 & 0.0 & 14.9 & 17514.7 & 224.7 & 4691.2 & 243.4 & 885.7 & 1193.7 & 4684.1 & 1657.4 \\
\hline 375 & 310.0 & 224.4 & 19046.3 & 1483.5 & 10323.7 & 1581.2 & 838.1 & 601.4 & 5258.6 & 2179.5 \\
\hline
\end{tabular}


Table S.5 Integrated Raman peak areas of uranyl and sulfate species versus temperature for Solution $5\left[0.530 \mathrm{~mol} \cdot \mathrm{kg}^{-1} \mathrm{UO}_{2} \mathrm{SO}_{4}+\right.$ $0.590 \mathrm{~mol} \cdot \mathrm{kg}^{-1} \mathrm{H}_{2} \mathrm{SO}_{4}$ ], in arbitrary units

\begin{tabular}{|c|c|c|c|c|c|c|c|c|c|c|}
\hline$T /{ }^{\circ} \mathrm{C}$ & $\mathrm{UO}_{2}{ }^{2+}$ & St. Error & $\mathrm{UO}_{2} \mathrm{SO}_{4}{ }^{0}$ & St. Error & $\mathrm{UO}_{2}\left(\mathrm{SO}_{4}\right)_{2}{ }^{2-}$ & St. Error & $\mathrm{SO}_{4}^{2-}$ & St. Error & $\mathrm{HSO}_{4}^{-}$ & St. Error \\
\hline 25 & 2199.9 & 89.7 & 7087.4 & 73.8 & 18378.2 & 250.3 & 2494.1 & 2331.8 & 4756.3 & 2140.7 \\
\hline 75 & 847.1 & 179.5 & 5753.9 & 169.6 & 20766.5 & 256.0 & 5217.4 & 5838.9 & 4637.8 & 9216.4 \\
\hline 125 & 1244.9 & 314.8 & 6389.8 & 133.1 & 19069.2 & 409.1 & 1678.1 & 13806.0 & 5491.3 & 6426.3 \\
\hline 175 & 1215.1 & 330.2 & 7082.1 & 161.2 & 16113.3 & 704.2 & 2191.0 & 4414.0 & 5490.8 & 4026.6 \\
\hline 225 & 870.5 & 77.2 & 6922.4 & 68.1 & 11960.3 & 1059.7 & 1835.5 & 3472.5 & 4308.0 & 1608.4 \\
\hline 275 & 1043.0 & 407.5 & 10070.8 & 1411.4 & 6464.9 & 1426.3 & 1339.4 & 1389.6 & 4081.9 & 1706.0 \\
\hline 325 & 112.8 & 242.2 & 10054.1 & 1192.0 & 10155.0 & 1013.8 & 786.6 & 1347.1 & 3423.5 & 642.4 \\
\hline 350 & 83.0 & 205.9 & 10814.8 & 597.4 & 5758.2 & 393.8 & 507.3 & 806.9 & 2500.5 & 673.3 \\
\hline 375 & 55.4 & 142.7 & 8257.1 & 356.2 & 4626.0 & 276.7 & 413.4 & 520.1 & 1352.9 & 516.0 \\
\hline
\end{tabular}

Table S.6 Integrated Raman peak areas of uranyl and sulfate species versus temperature for Solution $6\left[0.326 \mathrm{~mol} \cdot \mathrm{kg}^{-1} \mathrm{UO}_{2} \mathrm{SO}_{4}+\right.$ $\left.0.363 \mathrm{~mol} \cdot \mathrm{kg}^{-1} \mathrm{H}_{2} \mathrm{SO}_{4}\right]$, in arbitrary units

\begin{tabular}{ccccccccccc}
\hline$T /{ }^{\circ} \mathrm{C}$ & $\mathrm{UO}_{2}{ }^{2+}$ & St. Error & $\mathrm{UO}_{2} \mathrm{SO}_{4}{ }^{0}$ & St. Error & $\mathrm{UO}_{2}\left(\mathrm{SO}_{4}\right)_{2}{ }^{2-}$ & St. Error & $\mathrm{SO}_{4}{ }^{2-}$ & St. Error & $\mathrm{HSO}_{4}{ }^{-}$ & $\mathrm{St}^{2}$ Error \\
\hline 25 & 2143.6 & 97.6 & 6185.2 & 76.1 & 15478.3 & 214.6 & 1469.2 & 996.7 & 4146.9 & 2076.1 \\
75 & 1245.5 & 228.4 & 6993.4 & 185.9 & 23269.9 & 242.5 & 2540.0 & 8148.7 & 5488.6 & 8559.3 \\
125 & 1465.2 & 443.8 & 7429.3 & 181.1 & 20511.0 & 633.4 & 2637.4 & 12774.5 & 6107.7 & 9791.2 \\
175 & 1482.7 & 559.2 & 7747.3 & 262.2 & 18147.6 & 1217.1 & 1128.9 & 10216.1 & 7809.3 & 8364.8 \\
225 & 1209.1 & 148.8 & 7711.7 & 121.0 & 13565.0 & 1960.4 & 1214.5 & 3996.5 & 6398.9 & 3787.6 \\
275 & 489.1 & 327.3 & 12076.0 & 1452.0 & 5829.4 & 1605.9 & 560.7 & 2996.3 & 6187.7 & 1102.7 \\
325 & 0.0 & 237.7 & 11574.2 & 1809.5 & 7141.5 & 1698.0 & 515.3 & 288.0 & 5164.1 & 832.3 \\
350 & 0.0 & 82.7 & 8919.1 & 286.9 & 4571.9 & 209.6 & 524.9 & 565.9 & 3094.7 & 1263.5 \\
\hline
\end{tabular}


Table S.7 Integrated Raman peak areas of uranyl and sulfate species versus temperature for Solution $7\left[0.828 \mathrm{~mol} \cdot \mathrm{kg}^{-1} \mathrm{UO}_{2} \mathrm{SO}_{4}+\right.$ $1.286 \mathrm{~mol} \cdot \mathrm{kg}^{-1} \mathrm{H}_{2} \mathrm{SO}_{4}$ ], in arbitrary units

\begin{tabular}{|c|c|c|c|c|c|c|c|c|c|c|}
\hline$T /{ }^{\circ} \mathrm{C}$ & $\mathrm{UO}_{2}{ }^{2+}$ & St. Error & $\mathrm{UO}_{2} \mathrm{SO}_{4}{ }^{0}$ & St. Error & $\mathrm{UO}_{2}\left(\mathrm{SO}_{4}\right)_{2}{ }^{2-}$ & St. Error & $\mathrm{SO}_{4}^{2-}$ & St. Error & $\mathrm{HSO}_{4}^{-}$ & St. Error \\
\hline 25 & 3916.8 & 156.8 & 11751.6 & 97.3 & 32981.6 & 377.8 & 4547.1 & 3073.0 & 9955.1 & 2249.3 \\
\hline 75 & 2264.2 & 176.7 & 10271.1 & 101.0 & 35875.4 & 502.5 & 5157.8 & 18352.1 & 11295.5 & 14530.2 \\
\hline 125 & 2947.5 & 406.0 & 11778.0 & 99.4 & 30762.1 & 392.5 & 1862.8 & 37432.2 & 12691.8 & 9693.9 \\
\hline 175 & 2868.0 & 652.6 & 14052.5 & 1226.2 & 24540.3 & 1075.9 & 5593.4 & 1883.1 & 13409.5 & 4454.4 \\
\hline 225 & 1087.7 & 266.9 & 22386.1 & 1014.7 & 15987.9 & 1930.8 & 3454.3 & 3003.5 & 9600.0 & 3587.2 \\
\hline 275 & 1686.0 & 521.2 & 23837.5 & 1978.5 & 13646.8 & 2170.7 & 2560.6 & 3472.2 & 12878.8 & 3635.7 \\
\hline 325 & 14.5 & 132.0 & 22354.6 & 3801.6 & 15723.7 & 3736.4 & 1673.2 & 2159.4 & 7077.5 & 1703.5 \\
\hline 350 & 53.0 & 212.8 & 21566.3 & 2462.0 & 14935.2 & 2462.6 & 1270.5 & 1464.8 & 8486.3 & 2131.0 \\
\hline 375 & 0.0 & 47.2 & 22615.9 & 1449.0 & 10925.5 & 1428.8 & 998.1 & 1566.1 & 8916.7 & 4624.8 \\
\hline
\end{tabular}

Table S.8 Integrated Raman peak areas of uranyl and sulfate species versus temperature for Solution $8\left[0.536 \mathrm{~mol} \cdot \mathrm{kg}^{-1} \mathrm{UO}_{2} \mathrm{SO}_{4}+\right.$ $\left.0.833 \mathrm{~mol} \cdot \mathrm{kg}^{-1} \mathrm{H}_{2} \mathrm{SO}_{4}\right]$, in arbitrary units

\begin{tabular}{|c|c|c|c|c|c|c|c|c|c|c|}
\hline$T /{ }^{\circ} \mathrm{C}$ & $\mathrm{UO}_{2}{ }^{2+}$ & St. Error & $\mathrm{UO}_{2} \mathrm{SO}_{4}{ }^{0}$ & St. Error & $\mathrm{UO}_{2}\left(\mathrm{SO}_{4}\right)_{2}{ }^{2-}$ & St. Error & $\mathrm{SO}_{4}^{2-}$ & St. Error & $\mathrm{HSO}_{4}^{-}$ & St. Error \\
\hline 25 & 1999.4 & 120.7 & 5360.9 & 67.1 & 18821.3 & 184.1 & 1893.6 & 3813.6 & 6000.0 & 1782.6 \\
\hline 75 & 739.1 & 148.9 & 5549.1 & 142.4 & 19869.9 & 442.5 & 4795.2 & 6100.4 & 6737.2 & 9677.6 \\
\hline 125 & 1248.9 & 266.6 & 6399.4 & 103.2 & 17078.1 & 336.2 & 2119.8 & 7157.2 & 8099.4 & 7762.4 \\
\hline 175 & 1400.7 & 272.7 & 7375.1 & 105.3 & 15419.4 & 609.0 & 1681.4 & 4838.2 & 7789.5 & 3501.9 \\
\hline 225 & 1526.0 & 114.4 & 8369.6 & 87.9 & 10198.2 & 1343.0 & 1633.2 & 2361.2 & 7724.6 & 2695.9 \\
\hline 275 & 473.7 & 270.2 & 14451.1 & 1005.8 & 4743.1 & 942.7 & 741.3 & 2105.8 & 5535.4 & 1282.4 \\
\hline 325 & 0.0 & 25.6 & 12757.6 & 979.8 & 7870.4 & 951.5 & 608.3 & 1377.9 & 4865.4 & 737.0 \\
\hline 350 & 0.0 & 22.8 & 13492.4 & 489.0 & 5320.1 & 470.7 & 451.2 & 398.7 & 2884.7 & 935.2 \\
\hline 375 & 87.0 & 161.0 & 10836.9 & 596.2 & 6651.7 & 621.8 & 376.3 & 488.9 & 3069.5 & 922.1 \\
\hline
\end{tabular}


Table S.9 Integrated Raman peak areas of uranyl and sulfate species versus temperature for Solution $9\left[0.332 \mathrm{~mol} \cdot \mathrm{kg}^{-1} \mathrm{UO}_{2} \mathrm{SO}_{4}+\right.$ $0.516 \mathrm{~mol} \cdot \mathrm{kg}^{-1} \mathrm{H}_{2} \mathrm{SO}_{4}$ ], in arbitrary units

\begin{tabular}{|c|c|c|c|c|c|c|c|c|c|c|}
\hline$T /{ }^{\circ} \mathrm{C}$ & $\mathrm{UO}_{2}{ }^{2+}$ & St. Error & $\mathrm{UO}_{2} \mathrm{SO}_{4}{ }^{0}$ & St. Error & $\mathrm{UO}_{2}\left(\mathrm{SO}_{4}\right)_{2}{ }^{2-}$ & St. Error & $\mathrm{SO}_{4}^{2-}$ & St. Error & $\mathrm{HSO}_{4}^{-}$ & St. Error \\
\hline 25 & 2499.9 & 153.4 & 6846.5 & 92.4 & 22817.0 & 251.3 & 3172.1 & 1408.0 & 6974.0 & 2643.5 \\
\hline 75 & 1236.6 & 203.3 & 6687.2 & 173.5 & 24203.2 & 619.5 & 3141.5 & 14817.1 & 8208.3 & 9938.5 \\
\hline 125 & 1577.5 & 434.4 & 7669.7 & 185.3 & 20846.5 & 575.9 & 2755.6 & 11504.2 & 9369.8 & 8969.7 \\
\hline 175 & 1708.4 & 497.8 & 8370.9 & 235.4 & 18075.3 & 1078.4 & 1709.4 & 9132.6 & 9821.1 & 5051.1 \\
\hline 225 & 928.7 & 321.7 & 9530.6 & 1217.0 & 12671.5 & 2544.2 & 1104.2 & 2360.5 & 6856.8 & 3042.6 \\
\hline 275 & 397.4 & 219.0 & 15887.6 & 1102.3 & 6361.0 & 1325.8 & 934.6 & 3977.4 & 8596.8 & 2444.6 \\
\hline 325 & 0.0 & 25.9 & 14826.2 & 844.8 & 2563.3 & 2536.2 & 801.8 & 1137.2 & 5110.6 & 844.8 \\
\hline 350 & 0.0 & 33.0 & 15347.4 & 1971.6 & 530.5 & 503.0 & 583.6 & 1011.1 & 6347.4 & 1971.6 \\
\hline 375 & 0.0 & 21.1 & 10032.7 & 1726.8 & 389.6 & 377.8 & 416.8 & 589.3 & 3383.9 & 1726.8 \\
\hline
\end{tabular}

Table S.10 Integrated Raman peak areas of uranyl and sulfate species versus temperature for Solution $10\left[0.855 \mathrm{~mol} \cdot \mathrm{kg}^{-1} \mathrm{UO}_{2} \mathrm{SO}_{4}+\right.$ $\left.2.036 \mathrm{~mol} \cdot \mathrm{kg}^{-1} \mathrm{H}_{2} \mathrm{SO}_{4}\right]$, in arbitrary units

\begin{tabular}{|c|c|c|c|c|c|c|c|c|c|c|}
\hline$T /{ }^{\circ} \mathrm{C}$ & $\mathrm{UO}_{2}^{2+}$ & St. Error & $\mathrm{UO}_{2} \mathrm{SO}_{4}{ }^{0}$ & St. Error & $\mathrm{UO}_{2}\left(\mathrm{SO}_{4}\right)_{2}{ }^{2-}$ & St. Error & $\mathrm{SO}_{4}{ }^{2-}$ & St. Error & $\mathrm{HSO}_{4}^{-}$ & St. Error \\
\hline 25 & 3843.6 & 245.1 & 9208.1 & 98.9 & 34135.7 & 507.2 & 5855.8 & 17449.0 & 15020.2 & 3701.5 \\
\hline 75 & 2127.3 & 227.4 & 9193.5 & 98.4 & 36178.2 & 445.2 & 9555.4 & 13142.0 & 13987.8 & 18790.7 \\
\hline 125 & 2001.5 & 274.0 & 11422.3 & 99.1 & 29089.5 & 278.2 & 6449.6 & 8048.8 & 14925.7 & 10560.0 \\
\hline 175 & 2567.2 & 769.2 & 13639.1 & 1346.2 & 18787.7 & 1168.6 & 5359.3 & 8886.4 & 19596.9 & 4375.7 \\
\hline 225 & 3801.4 & 727.6 & 15688.0 & 1843.1 & 17814.1 & 3122.5 & 2996.2 & 10239.5 & 15566.6 & 10078.1 \\
\hline 275 & 1839.1 & 882.8 & 23845.3 & 2427.5 & 11834.1 & 1928.6 & 2168.2 & 9796.9 & 18743.5 & 6285.2 \\
\hline 325 & 0.0 & 55.6 & 26835.4 & 1877.6 & 12460.6 & 1829.2 & 1188.3 & 1740.0 & 16415.4 & 1816.6 \\
\hline 350 & 0.0 & 66.8 & 25601.7 & 2390.5 & 12535.6 & 2348.0 & 1047.1 & 2840.6 & 15622.4 & 8280.4 \\
\hline 375 & 0.0 & 28.8 & 23929.4 & 27341.2 & 10715.7 & 27229.8 & 791.4 & 1440.7 & 13332.6 & 1414.8 \\
\hline
\end{tabular}


Table S.11 Integrated Raman peak areas of uranyl and sulfate species versus temperature for Solution $11\left[0.567 \mathrm{~mol} \cdot \mathrm{kg}^{-1} \mathrm{UO}_{2} \mathrm{SO}_{4}+\right.$ $1.349 \mathrm{~mol} \cdot \mathrm{kg}^{-1} \mathrm{H}_{2} \mathrm{SO}_{4}$ ], in arbitrary units

\begin{tabular}{|c|c|c|c|c|c|c|c|c|c|c|}
\hline$T /{ }^{\circ} \mathrm{C}$ & $\mathrm{UO}_{2}{ }^{2+}$ & St. Error & $\mathrm{UO}_{2} \mathrm{SO}_{4}{ }^{0}$ & St. Error & $\mathrm{UO}_{2}\left(\mathrm{SO}_{4}\right)_{2}{ }^{2-}$ & St. Error & $\mathrm{SO}_{4}^{2-}$ & St. Error & $\mathrm{HSO}_{4}^{-}$ & St. Error \\
\hline 25 & 2254.2 & 136.3 & 4560.1 & 58.3 & 19732.9 & 172.8 & 5055.6 & 10694.7 & 8063.5 & 2948.2 \\
\hline 75 & 969.9 & 116.8 & 5502.5 & 82.1 & 21100.1 & 301.4 & 8645.8 & 3630.5 & 8144.2 & 7544.4 \\
\hline 125 & 1360.0 & 202.8 & 6615.6 & 73.0 & 17314.0 & 252.7 & 3364.0 & 4902.3 & 9107.3 & 5261.7 \\
\hline 175 & 1546.6 & 263.0 & 7772.8 & 113.8 & 13148.4 & 510.9 & 2261.0 & 3011.7 & 9926.6 & 3734.0 \\
\hline 225 & 523.1 & 155.4 & 15026.3 & 675.3 & 7689.3 & 1294.1 & 1699.3 & 2198.0 & 9467.8 & 3165.6 \\
\hline 275 & 35.6 & 104.9 & 19348.0 & 569.3 & 3879.6 & 675.2 & 1283.3 & 1181.7 & 11600.0 & 4587.0 \\
\hline 325 & 362.2 & 356.9 & 14440.5 & 1334.5 & 6143.3 & 1152.9 & 288.4 & 807.9 & 6698.5 & 1414.8 \\
\hline 350 & 0.0 & 19.4 & 14308.7 & 633.3 & 6489.8 & 622.6 & 504.7 & 1152.3 & 5293.3 & 1333.0 \\
\hline 375 & 253.9 & 220.2 & 12466.4 & 756.9 & 6193.2 & 740.3 & 245.9 & 338.6 & 4302.2 & 1746.6 \\
\hline
\end{tabular}

Table S.12 Integrated Raman peak areas of uranyl and sulfate species versus temperature for Solution $12\left[0.338 \mathrm{~mol} \cdot \mathrm{kg}^{-1} \mathrm{UO}_{2} \mathrm{SO}_{4}+\right.$ $\left.0.805 \mathrm{~mol} \cdot \mathrm{kg}^{-1} \mathrm{H}_{2} \mathrm{SO}_{4}\right]$, in arbitrary units

\begin{tabular}{ccccccccccc}
\hline$T /{ }^{\circ} \mathrm{C}$ & $\mathrm{UO}_{2}{ }^{2+}$ & St. Error & $\mathrm{UO}_{2} \mathrm{SO}_{4}{ }^{0}$ & St. Error & $\mathrm{UO}_{2}\left(\mathrm{SO}_{4}\right)_{2}{ }^{2-}$ & St. Error & $\mathrm{SO}_{4}{ }^{2-}$ & St. Error & $\mathrm{HSO}_{4}{ }^{-}$ & $\mathrm{St}^{-}$Error \\
\hline 25 & 2288.8 & 156.4 & 6406.7 & 87.1 & 25966.1 & 272.4 & 3959.6 & 805.7 & 9809.9 & 4471.0 \\
75 & 1073.8 & 203.3 & 6631.7 & 178.7 & 25949.0 & 556.7 & 3704.6 & 21598.4 & 12290.1 & 9602.9 \\
125 & 1179.5 & 200.7 & 4818.4 & 72.7 & 12676.6 & 257.9 & 2377.0 & 5232.9 & 10129.0 & 5954.7 \\
175 & 1215.8 & 327.7 & 5551.9 & 149.7 & 12390.0 & 684.9 & 1682.6 & 4451.9 & 10577.6 & 4648.4 \\
225 & 1388.0 & 109.2 & 6241.9 & 82.6 & 10219.4 & 1344.3 & 1458.1 & 2390.1 & 8585.8 & 2715.5 \\
275 & 800.0 & 429.4 & 9915.9 & 1245.5 & 5100.0 & 880.5 & 995.4 & 1439.7 & 7000.0 & 2308.5 \\
325 & 0.0 & 19.4 & 13506.5 & 369.6 & 3571.8 & 394.9 & 489.0 & 866.5 & 7219.1 & 777.8 \\
350 & 0.0 & 11.5 & 12657.8 & 300.1 & 3748.5 & 295.3 & 447.5 & 940.9 & 5949.0 & 1651.1 \\
375 & 260.6 & 172.3 & 10142.5 & 498.9 & 3847.8 & 358.8 & 349.8 & 1384.6 & 4214.3 & 2731.4 \\
\hline
\end{tabular}


Table S.13 Molalities of aqueous species versus temperature for Solution $1\left[0.702 \mathrm{~mol} \cdot \mathrm{kg}^{-1} \mathrm{UO}_{2} \mathrm{SO}_{4}+0.375 \mathrm{~mol} \cdot \mathrm{kg}^{-1} \mathrm{H}_{2} \mathrm{SO}_{4}\right]$, derived from experimental Raman data

\begin{tabular}{cccccccc}
\hline$T /{ }^{\circ} \mathrm{C}$ & \multicolumn{7}{c}{ Molality / mol $\cdot \mathrm{kg}^{-1}$} \\
\cline { 2 - 7 } & $\mathrm{UO}_{2}{ }^{2+}$ & $\mathrm{UO}_{2} \mathrm{SO}_{4}{ }^{0}$ & $\mathrm{UO}_{2}\left(\mathrm{SO}_{4}\right)_{2}{ }^{2-}$ & $\mathrm{HSO}_{4}{ }^{-}$ & $\mathrm{H}^{+}$ & $\mathrm{SO}_{4}{ }^{2-}$ & $\mathrm{Strength}^{-}$ \\
\hline 25 & $0.178 \pm 0.021$ & $0.369 \pm 0.081$ & $0.154 \pm 0.022$ & $0.251 \pm 0.200$ & $0.500 \pm 0.200$ & $(3.39 \pm 3.03) \cdot 10^{-2}$ & 1.11 \\
75 & $0.094 \pm 0.003$ & $0.321 \pm 0.018$ & $0.287 \pm 0.018$ & $0.320 \pm 1.005$ & $0.431 \pm 1.005$ & $(1.59 \pm 6.23) \cdot 10^{-2}$ & 1.17 \\
125 & $0.084 \pm 0.004$ & $0.338 \pm 0.022$ & $0.280 \pm 0.020$ & $0.308 \pm 0.412$ & $0.443 \pm 0.412$ & $(5.49 \pm 8.96) \cdot 10^{-3}$ & 1.11 \\
175 & $0.084 \pm 0.004$ & $0.362 \pm 0.023$ & $0.256 \pm 0.020$ & $0.332 \pm 0.301$ & $0.419 \pm 0.301$ & $(1.52 \pm 1.76) \cdot 10^{-3}$ & 1.06 \\
225 & $0.064 \pm 0.006$ & $0.409 \pm 0.060$ & $0.228 \pm 0.044$ & $0.229 \pm 0.109$ & $0.522 \pm 0.109$ & $(2.14 \pm 1.12) \cdot 10^{-4}$ & 0.96 \\
275 & $0.059 \pm 0.008$ & $0.499 \pm 0.110$ & $0.144 \pm 0.039$ & $0.445 \pm 0.148$ & $0.306 \pm 0.148$ & $(2.27 \pm 1.34) \cdot 10^{-4}$ & 0.78 \\
325 & $0.018 \pm 0.001$ & $0.535 \pm 0.055$ & $0.148 \pm 0.015$ & $0.366 \pm 0.068$ & $0.385 \pm 0.068$ & $(2.34 \pm 0.62) \cdot 10^{-4}$ & 0.71 \\
\hline
\end{tabular}

Table S.14 Molalities of aqueous species versus temperature for Solution $2\left[0.514 \mathrm{~mol} \cdot \mathrm{kg}^{-1} \mathrm{UO}_{2} \mathrm{SO}_{4}+0.275 \mathrm{~mol} \cdot \mathrm{kg}^{-1} \mathrm{H}_{2} \mathrm{SO}_{4}\right]$, derived from experimental Raman data

\begin{tabular}{cccccccc}
\hline$T /{ }^{\circ} \mathrm{C}$ & \multicolumn{7}{c}{ Molality / mol $\cdot \mathrm{kg}^{-1}$} \\
\cline { 2 - 7 } & $\mathrm{UO}_{2}{ }^{2+}$ & $\mathrm{UO}_{2} \mathrm{SO}_{4}{ }^{0}$ & $\mathrm{UO}_{2}\left(\mathrm{SO}_{4}\right)_{2}{ }^{2-}$ & $\mathrm{HSO}_{4}{ }^{-}$ & $\mathrm{H}^{+}$ & $\mathrm{SO}_{4}{ }^{2-}$ & $\mathrm{Strength}^{-}$ \\
\hline 25 & $0.109 \pm 0.027$ & $0.263 \pm 0.123$ & $0.142 \pm 0.040$ & $0.259 \pm 0.178$ & $0.291 \pm 0.179$ & $(5.38 \pm 4.97) \cdot 10^{-2}$ & 0.88 \\
75 & $0.048 \pm 0.003$ & $0.241 \pm 0.019$ & $0.224 \pm 0.017$ & $0.242 \pm 0.708$ & $0.309 \pm 0.708$ & $(1.49 \pm 5.56) \cdot 10^{-2}$ & 0.85 \\
125 & $0.035 \pm 0.002$ & $0.253 \pm 0.018$ & $0.227 \pm 0.017$ & $0.337 \pm 0.681$ & $0.214 \pm 0.681$ & $(1.07 \pm 4.03) \cdot 10^{-2}$ & 0.82 \\
175 & $0.061 \pm 0.004$ & $0.259 \pm 0.022$ & $0.194 \pm 0.020$ & $0.310 \pm 0.432$ & $0.241 \pm 0.432$ & $(2.12 \pm 4.82) \cdot 10^{-3}$ & 0.79 \\
225 & $0.050 \pm 0.003$ & $0.303 \pm 0.023$ & $0.161 \pm 0.026$ & $0.325 \pm 0.305$ & $0.225 \pm 0.305$ & $(5.90 \pm 9.76) \cdot 10^{-4}$ & 0.70 \\
275 & $0.065 \pm 0.003$ & $0.339 \pm 0.023$ & $0.110 \pm 0.013$ & $0.235 \pm 0.127$ & $0.315 \pm 0.128$ & $(1.00 \pm 0.68) \cdot 10^{-4}$ & 0.63 \\
325 & 0 & $0.472 \pm 0.055$ & $0.043 \pm 0.010$ & $0.240 \pm 0.070$ & $0.311 \pm 0.071$ & $(8.24 \pm 3.10) \cdot 10^{-5}$ & 0.36 \\
\hline
\end{tabular}


Table S.15 Molalities of aqueous species versus temperature for Solution $3\left[0.317 \mathrm{~mol} \cdot \mathrm{kg}^{-1} \mathrm{UO}_{2} \mathrm{SO}_{4}+0.169 \mathrm{~mol} \cdot \mathrm{kg}^{-1} \mathrm{H}_{2} \mathrm{SO}_{4}\right]$, derived from experimental Raman data

\begin{tabular}{cccccccc}
\hline$T /{ }^{\circ} \mathrm{C}$ & \multicolumn{7}{c}{ Molality / mol $\cdot \mathrm{kg}^{-1}$} \\
\cline { 2 - 7 } & $\mathrm{UO}_{2}{ }^{2+}$ & $\mathrm{UO}_{2} \mathrm{SO}_{4}{ }^{0}$ & $\mathrm{UO}_{2}\left(\mathrm{SO}_{4}\right)_{2}{ }^{2-}$ & $\mathrm{HSO}_{4}{ }^{-}$ & $\mathrm{H}^{+}$ & $\mathrm{SO}_{4}{ }^{2-}$ & $\mathrm{Strength}^{-}$ \\
\hline 25 & $0.082 \pm 0.011$ & $0.162 \pm 0.042$ & $0.073 \pm 0.012$ & $0.153 \pm 0.153$ & $0.186 \pm 0.153$ & $(4.37 \pm 5.66) \cdot 10^{-2}$ & 0.57 \\
75 & $0.026 \pm 0.002$ & $0.154 \pm 0.015$ & $0.137 \pm 0.012$ & $0.121 \pm 0.598$ & $0.218 \pm 0.598$ & $(9.04 \pm 51.0) \cdot 10^{-3}$ & 0.51 \\
125 & $0.033 \pm 0.003$ & $0.153 \pm 0.015$ & $0.131 \pm 0.014$ & $0.162 \pm 0.363$ & $0.177 \pm 0.363$ & $(4.85 \pm 14.7) \cdot 10^{-3}$ & 0.51 \\
175 & $0.036 \pm 0.003$ & $0.158 \pm 0.017$ & $0.123 \pm 0.016$ & $0.214 \pm 0.295$ & $0.125 \pm 0.295$ & $(2.19 \pm 5.99) \cdot 10^{-3}$ & 0.49 \\
225 & $0.031 \pm 0.002$ & $0.190 \pm 0.017$ & $0.096 \pm 0.017$ & $0.166 \pm 0.229$ & $0.173 \pm 0.229$ & $(2.91 \pm 5.56) \cdot 10^{-4}$ & 0.42 \\
275 & $0.021 \pm 0.003$ & $0.210 \pm 0.050$ & $0.086 \pm 0.017$ & $0.206 \pm 0.169$ & $0.133 \pm 0.169$ & $(1.45 \pm 2.20) \cdot 10^{-4}$ & 0.38 \\
325 & 0 & $0.242 \pm 0.066$ & $0.075 \pm 0.020$ & $0.152 \pm 0.071$ & $0.187 \pm 0.072$ & $(7.49 \pm 4.54) \cdot 10^{-5}$ & 0.32 \\
\hline
\end{tabular}

Table S.16 Molalities of aqueous species versus temperature for Solution $4\left[0.757 \mathrm{~mol} \cdot \mathrm{kg}^{-1} \mathrm{UO}_{2} \mathrm{SO}_{4}+0.842 \mathrm{~mol} \cdot \mathrm{kg}^{-1} \mathrm{H}_{2} \mathrm{SO}_{4}\right]$, derived from experimental Raman data

\begin{tabular}{|c|c|c|c|c|c|c|c|}
\hline \multirow[t]{2}{*}{$T /{ }^{\circ} \mathrm{C}$} & \multicolumn{7}{|c|}{ Molality / $\mathrm{mol} \cdot \mathrm{kg}^{-1}$} \\
\hline & $\mathrm{UO}_{2}{ }^{2+}$ & $\mathrm{UO}_{2} \mathrm{SO}_{4}{ }^{0}$ & $\mathrm{UO}_{2}\left(\mathrm{SO}_{4}\right)_{2}{ }^{2-}$ & $\mathrm{HSO}_{4}^{-}$ & $\mathrm{H}^{+}$ & $\mathrm{SO}_{4}^{2-}$ & $\begin{array}{c}\text { Ionic } \\
\text { Strength }\end{array}$ \\
\hline 25 & $0.149 \pm 0.005$ & $0.335 \pm 0.016$ & $0.273 \pm 0.016$ & $0.573 \pm 0.180$ & $1.111 \pm 0.180$ & $(2.77 \pm 1.00) \cdot 10^{-2}$ & 1.74 \\
\hline 75 & $0.107 \pm 0.004$ & $0.333 \pm 0.018$ & $0.317 \pm 0.020$ & $0.744 \pm 0.856$ & $0.939 \pm 0.856$ & $(1.36 \pm 1.99) \cdot 10^{-2}$ & 1.72 \\
\hline 125 & $0.110 \pm 0.004$ & $0.356 \pm 0.020$ & $0.292 \pm 0.019$ & $0.685 \pm 0.459$ & $0.999 \pm 0.459$ & $(6.57 \pm 5.36) \cdot 10^{-3}$ & 1.66 \\
\hline 175 & $0.099 \pm 0.004$ & $0.415 \pm 0.024$ & $0.244 \pm 0.018$ & $0.757 \pm 0.330$ & $0.927 \pm 0.330$ & $(1.88 \pm 1.07) \cdot 10^{-3}$ & 1.53 \\
\hline 225 & $0.072 \pm 0.005$ & $0.514 \pm 0.055$ & $0.171 \pm 0.029$ & $0.480 \pm 0.291$ & $1.204 \pm 0.291$ & $(2.32 \pm 1.52) \cdot 10^{-4}$ & 1.33 \\
\hline 275 & $0.010 \pm 0.001$ & $0.626 \pm 0.042$ & $0.121 \pm 0.012$ & $0.477 \pm 0.218$ & $1.206 \pm 0.218$ & $(7.78 \pm 3.86) \cdot 10^{-5}$ & 1.10 \\
\hline 325 & 0 & $0.597 \pm 0.190$ & $0.156 \pm 0.053$ & $0.580 \pm 0.266$ & $1.104 \pm 0.266$ & $(2.32 \pm 1.21) \cdot 10^{-4}$ & 1.16 \\
\hline 350 & 0 & $0.701 \pm 0.005$ & $0.056 \pm 0.187$ & $0.520 \pm 0.187$ & $1.164 \pm 0.187$ & $(8.45 \pm 3.39) \cdot 10^{-4}$ & 0.96 \\
\hline 375 & $0.015 \pm 0.001$ & $0.638 \pm 0.075$ & $0.104 \pm 0.019$ & $0.489 \pm 0.215$ & $1.195 \pm 0.215$ & $(1.15 \pm 0.55) \cdot 10^{-4}$ & 1.08 \\
\hline
\end{tabular}


Table S.17 Molalities of aqueous species versus temperature for Solution 5 [0.530 mol $\left.\cdot \mathrm{kg}^{-1} \mathrm{UO}_{2} \mathrm{SO}_{4}+0.590 \mathrm{~mol} \cdot \mathrm{kg}^{-1} \mathrm{H}_{2} \mathrm{SO}_{4}\right]$, derived from experimental Raman data

\begin{tabular}{cccccccc}
\hline$T /{ }^{\circ} \mathrm{C}$ & \multicolumn{7}{c}{ Molality / mol $\cdot \mathrm{kg}^{-1}$} \\
\cline { 2 - 7 } & $\mathrm{UO}_{2}{ }^{2+}$ & $\mathrm{UO}_{2} \mathrm{SO}_{4}{ }^{0}$ & $\mathrm{UO}_{2}\left(\mathrm{SO}_{4}\right)_{2}{ }^{2-}$ & $\mathrm{HSO}_{4}{ }^{-}$ & $\mathrm{H}^{+}$ & $\mathrm{SO}_{4}{ }^{2-}$ & $\mathrm{Strength}^{-}$ \\
\hline 25 & $0.108 \pm 0.005$ & $0.237 \pm 0.014$ & $0.185 \pm 0.013$ & $0.442 \pm 0.201$ & $0.737 \pm 0.203$ & $(3.24 \pm 1.74) \cdot 10^{-2}$ & 1.24 \\
75 & $0.050 \pm 0.003$ & $0.231 \pm 0.016$ & $0.250 \pm 0.018$ & $0.516 \pm 1.026$ & $0.663 \pm 1.026$ & $(1.32 \pm 3.34) \cdot 10^{-2}$ & 1.22 \\
125 & $0.069 \pm 0.004$ & $0.243 \pm 0.017$ & $0.218 \pm 0.017$ & $0.580 \pm 0.680$ & $0.599 \pm 0.681$ & $(7.86 \pm 12.9) \cdot 10^{-3}$ & 1.18 \\
175 & $0.069 \pm 0.004$ & $0.274 \pm 0.020$ & $0.187 \pm 0.017$ & $0.590 \pm 0.435$ & $0.589 \pm 0.436$ & $(1.96 \pm 2.06) \cdot 10^{-3}$ & 1.11 \\
225 & $0.057 \pm 0.003$ & $0.311 \pm 0.020$ & $0.162 \pm 0.019$ & $0.538 \pm 0.205$ & $0.641 \pm 0.206$ & $(4.25 \pm 2.14) \cdot 10^{-4}$ & 1.03 \\
275 & $0.060 \pm 0.007$ & $0.394 \pm 0.076$ & $0.076 \pm 0.020$ & $0.444 \pm 0.214$ & $0.735 \pm 0.215$ & $(1.01 \pm 0.57) \cdot 10^{-4}$ & 0.86 \\
325 & $0.007 \pm 0.001$ & $0.402 \pm 0.066$ & $0.122 \pm 0.019$ & $0.380 \pm 0.106$ & $0.799 \pm 0.109$ & $(1.45 \pm 0.46) \cdot 10^{-4}$ & 0.85 \\
350 & 0 & $0.453 \pm 0.008$ & $0.072 \pm 0.085$ & $0.290 \pm 0.089$ & $0.899 \pm 0.089$ & $(3.89 \pm 1.24) \cdot 10^{-4}$ & 0.75 \\
375 & $0.004 \pm 0.001$ & $0.450 \pm 0.038$ & $0.076 \pm 0.008$ & $0.205 \pm 0.081$ & $0.974 \pm 0.085$ & $(4.07 \pm 1.67) \cdot 10^{-5}$ & 0.75 \\
\hline
\end{tabular}

Table S.18 Molalities of aqueous species versus temperature for Solution $6\left[0.326 \mathrm{~mol} \cdot \mathrm{kg}^{-1} \mathrm{UO}_{2} \mathrm{SO}_{4}+0.363 \mathrm{~mol} \cdot \mathrm{kg}^{-1} \mathrm{H}_{2} \mathrm{SO}_{4}\right]$, derived from experimental Raman data

\begin{tabular}{cccccccc}
\hline$T /{ }^{\circ} \mathrm{C}$ & \multicolumn{7}{c}{ Molality / mol $\cdot \mathrm{kg}^{-1}$} \\
\cline { 2 - 7 } & $\mathrm{UO}_{2}{ }^{2+}$ & $\mathrm{UO}_{2} \mathrm{SO}_{4}{ }^{0}$ & $\mathrm{UO}_{2}\left(\mathrm{SO}_{4}\right)_{2}{ }^{2-}$ & $\mathrm{HSO}_{4}{ }^{-}$ & $\mathrm{H}^{+}$ & $\mathrm{SO}_{4}{ }^{2-}$ & $\mathrm{Strength}^{-}$ \\
\hline 25 & $0.073 \pm 0.003$ & $0.145 \pm 0.009$ & $0.109 \pm 0.007$ & $0.269 \pm 0.136$ & $0.457 \pm 0.137$ & $(3.05 \pm 1.81) \cdot 10^{-2}$ & 0.79 \\
75 & $0.038 \pm 0.002$ & $0.144 \pm 0.010$ & $0.144 \pm 0.010$ & $0.315 \pm 0.491$ & $0.412 \pm 0.492$ & $(1.25 \pm 2.46) \cdot 10^{-2}$ & 0.75 \\
125 & $0.044 \pm 0.003$ & $0.154 \pm 0.012$ & $0.128 \pm 0.011$ & $0.352 \pm 0.565$ & $0.374 \pm 0.565$ & $(5.95 \pm 13.1) \cdot 10^{-3}$ & 0.72 \\
175 & $0.046 \pm 0.003$ & $0.165 \pm 0.015$ & $0.116 \pm 0.013$ & $0.461 \pm 0.496$ & $0.266 \pm 0.496$ & $(2.66 \pm 5.74) \cdot 10^{-3}$ & 0.69 \\
225 & $0.043 \pm 0.003$ & $0.186 \pm 0.014$ & $0.098 \pm 0.016$ & $0.428 \pm 0.256$ & $0.298 \pm 0.256$ & $(5.59 \pm 5.86) \cdot 10^{-4}$ & 0.65 \\
275 & $0.016 \pm 0.002$ & $0.271 \pm 0.047$ & $0.039 \pm 0.012$ & $0.386 \pm 0.108$ & $0.341 \pm 0.109$ & $(1.24 \pm 0.53) \cdot 10^{-4}$ & 0.47 \\
325 & 0 & $0.275 \pm 0.060$ & $0.051 \pm 0.015$ & $0.341 \pm 0.107$ & $0.385 \pm 0.109$ & $(1.30 \pm 0.56) \cdot 10^{-4}$ & 0.47 \\
350 & 0 & $0.283 \pm 0.004$ & $0.044 \pm 0.114$ & $0.272 \pm 0.115$ & $0.454 \pm 0.115$ & $(2.79 \pm 1.38) \cdot 10^{-4}$ & 0.45 \\
\hline
\end{tabular}


Table S.19 Molalities of aqueous species versus temperature for Solution $7\left[0.828 \mathrm{~mol} \cdot \mathrm{kg}^{-1} \mathrm{UO}_{2} \mathrm{SO}_{4}+1.286 \mathrm{~mol} \cdot \mathrm{kg}^{-1} \mathrm{H}_{2} \mathrm{SO}_{4}\right]$, derived from experimental Raman data

\begin{tabular}{cccccccc}
\hline$T /{ }^{\circ} \mathrm{C}$ & \multicolumn{7}{c}{ Molality / mol $\cdot \mathrm{kg}^{-1}$} \\
\cline { 2 - 7 } & $\mathrm{UO}_{2}{ }^{2+}$ & $\mathrm{UO}_{2} \mathrm{SO}_{4}{ }^{0}$ & $\mathrm{UO}_{2}\left(\mathrm{SO}_{4}\right)^{2-}$ & $\mathrm{HSO}_{4}{ }^{-}$ & $\mathrm{H}^{+}$ & $\mathrm{SO}_{4}{ }^{2-}$ & $\mathrm{Strength}^{-}$ \\
\hline 25 & $0.173 \pm 0.005$ & $0.335 \pm 0.018$ & $0.300 \pm 0.018$ & $0.835 \pm 0.196$ & $1.737 \pm 0.196$ & $(2.07 \pm 0.58) \cdot 10^{-2}$ & 2.27 \\
75 & $0.112 \pm 0.004$ & $0.349 \pm 0.018$ & $0.366 \pm 0.023$ & $1.066 \pm 1.373$ & $1.506 \pm 1.373$ & $(9.50 \pm 15.0) \cdot 10^{-3}$ & 2.26 \\
125 & $0.141 \pm 0.005$ & $0.385 \pm 0.021$ & $0.302 \pm 0.020$ & $1.152 \pm 0.833$ & $1.421 \pm 0.833$ & $(8.84 \pm 8.75) \cdot 10^{-3}$ & 2.19 \\
175 & $0.135 \pm 0.009$ & $0.454 \pm 0.053$ & $0.238 \pm 0.023$ & $1.203 \pm 0.439$ & $1.369 \pm 0.439$ & $(2.32 \pm 1.14) \cdot 10^{-3}$ & 2.04 \\
225 & $0.046 \pm 0.002$ & $0.644 \pm 0.052$ & $0.138 \pm 0.020$ & $0.767 \pm 0.296$ & $1.806 \pm 0.296$ & $(2.77 \pm 1.18) \cdot 10^{-4}$ & 1.65 \\
275 & $0.067 \pm 0.005$ & $0.649 \pm 0.079$ & $0.112 \pm 0.021$ & $0.973 \pm 0.312$ & $1.599 \pm 0.312$ & $(1.54 \pm 0.59) \cdot 10^{-4}$ & 1.64 \\
325 & 0 & $0.683 \pm 0.157$ & $0.144 \pm 0.041$ & $0.600 \pm 0.226$ & $1.972 \pm 0.226$ & $(1.90 \pm 0.76) \cdot 10^{-4}$ & 1.58 \\
350 & 0 & $0.683 \pm 0.029$ & $0.142 \pm 0.239$ & $0.746 \pm 0.240$ & $1.826 \pm 0.240$ & $(1.91 \pm 0.68) \cdot 10^{-3}$ & 1.58 \\
375 & 0 & $0.723 \pm 0.073$ & $0.105 \pm 0.016$ & $0.791 \pm 0.422$ & $1.781 \pm 0.422$ & $(1.42 \pm 0.83) \cdot 10^{-4}$ & 1.50 \\
\hline
\end{tabular}

Table S.20 Molalities of aqueous species versus temperature for Solution $8\left[0.536 \mathrm{~mol} \cdot \mathrm{kg}^{-1} \mathrm{UO}_{2} \mathrm{SO}_{4}+0.833 \mathrm{~mol} \cdot \mathrm{kg}^{-1} \mathrm{H}_{2} \mathrm{SO}_{4}\right]$, derived from experimental Raman data

\begin{tabular}{|c|c|c|c|c|c|c|c|}
\hline \multirow[t]{2}{*}{$T /{ }^{\circ} \mathrm{C}$} & \multicolumn{7}{|c|}{ Molality $/ \mathrm{mol} \cdot \mathrm{kg}^{-1}$} \\
\hline & $\mathrm{UO}_{2}^{2+}$ & $\mathrm{UO}_{2} \mathrm{SO}_{4}{ }^{0}$ & $\mathrm{UO}_{2}\left(\mathrm{SO}_{4}\right)_{2}^{2-}$ & $\mathrm{HSO}_{4}^{-}$ & $\mathrm{H}^{+}$ & $\mathrm{SO}_{4}{ }^{2-}$ & $\begin{array}{c}\text { Ionic } \\
\text { Strength }\end{array}$ \\
\hline 25 & $0.112 \pm 0.005$ & $0.206 \pm 0.012$ & $0.217 \pm 0.015$ & $0.641 \pm 0.196$ & $1.025 \pm 0.199$ & $(3.02 \pm 1.11) \cdot 10^{-2}$ & 1.55 \\
\hline 75 & $0.046 \pm 0.002$ & $0.236 \pm 0.016$ & $0.254 \pm 0.019$ & $0.796 \pm 1.145$ & $0.870 \pm 1.145$ & $(1.34 \pm 2.61) \cdot 10^{-2}$ & 1.46 \\
\hline 125 & $0.073 \pm 0.004$ & $0.257 \pm 0.017$ & $0.206 \pm 0.016$ & $0.902 \pm 0.868$ & $0.763 \pm 0.868$ & $(1.05 \pm 1.57) \cdot 10^{-2}$ & 1.41 \\
\hline 175 & $0.078 \pm 0.004$ & $0.281 \pm 0.019$ & $0.177 \pm 0.015$ & $0.825 \pm 0.376$ & $0.841 \pm 0.378$ & $(2.12 \pm 1.37) \cdot 10^{-3}$ & 1.35 \\
\hline 225 & $0.087 \pm 0.005$ & $0.328 \pm 0.022$ & $0.120 \pm 0.018$ & $0.841 \pm 0.300$ & $0.824 \pm 0.302$ & $(5.75 \pm 2.98) \cdot 10^{-4}$ & 1.25 \\
\hline 275 & $0.022 \pm 0.002$ & $0.468 \pm 0.053$ & $0.046 \pm 0.010$ & $0.497 \pm 0.134$ & $1.168 \pm 0.139$ & $(7.68 \pm 2.33) \cdot 10^{-5}$ & 0.97 \\
\hline 325 & 0 & $0.452 \pm 0.053$ & $0.084 \pm 0.013$ & $0.479 \pm 0.101$ & $1.187 \pm 0.106$ & $(1.50 \pm 0.36) \cdot 10^{-4}$ & 1.00 \\
\hline 350 & 0 & $0.479 \pm 0.007$ & $0.057 \pm 0.096$ & $0.284 \pm 0.102$ & $1.381 \pm 0.102$ & $(3.82 \pm 1.35) \cdot 10^{-4}$ & 0.95 \\
\hline 375 & 0 & $0.448 \pm 0.043$ & $0.083 \pm 0.010$ & $0.352 \pm 0.114$ & $1.313 \pm 0.119$ & $(6.92 \pm 2.37) \cdot 10^{-5}$ & 1.01 \\
\hline
\end{tabular}


Table S.21 Molalities of aqueous species versus temperature for Solution 9 [0.332 mol $\left.\cdot \mathrm{kg}^{-1} \mathrm{UO}_{2} \mathrm{SO}_{4}+0.516 \mathrm{~mol} \cdot \mathrm{kg}^{-1} \mathrm{H}_{2} \mathrm{SO}_{4}\right]$, derived from experimental Raman data

\begin{tabular}{|c|c|c|c|c|c|c|c|}
\hline \multirow[t]{2}{*}{$T /{ }^{\circ} \mathrm{C}$} & \multicolumn{7}{|c|}{ Molality $/ \mathrm{mol} \cdot \mathrm{kg}^{-1}$} \\
\hline & $\mathrm{UO}_{2}{ }^{2+}$ & $\mathrm{UO}_{2} \mathrm{SO}_{4}{ }^{0}$ & $\mathrm{UO}_{2}\left(\mathrm{SO}_{4}\right)_{2}{ }^{2-}$ & $\mathrm{HSO}_{4}^{-}$ & $\mathrm{H}^{+}$ & $\mathrm{SO}_{4}{ }^{2-}$ & $\begin{array}{c}\text { Ionic } \\
\text { Strength }\end{array}$ \\
\hline 25 & $0.070 \pm 0.003$ & $0.131 \pm 0.008$ & $0.131 \pm 0.009$ & $0.370 \pm 0.143$ & $0.661 \pm 0.144$ & $(2.78 \pm 1.25) \cdot 10^{-2}$ & 0.97 \\
\hline 75 & $0.038 \pm 0.002$ & $0.141 \pm 0.009$ & $0.153 \pm 0.012$ & $0.480 \pm 0.582$ & $0.551 \pm 0.582$ & $(1.35 \pm 2.18) \cdot 10^{-2}$ & 0.92 \\
\hline 125 & $0.047 \pm 0.003$ & $0.157 \pm 0.012$ & $0.128 \pm 0.011$ & $0.532 \pm 0.511$ & $0.499 \pm 0.511$ & $(7.47 \pm 10.5) \cdot 10^{-3}$ & 0.88 \\
\hline 175 & $0.051 \pm 0.003$ & $0.170 \pm 0.014$ & $0.111 \pm 0.011$ & $0.555 \pm 0.290$ & $0.476 \pm 0.291$ & $(1.99 \pm 1.60) \cdot 10^{-3}$ & 0.84 \\
\hline 225 & $0.031 \pm 0.003$ & $0.215 \pm 0.037$ & $0.086 \pm 0.020$ & $0.430 \pm 0.213$ & $0.601 \pm 0.214$ & $(3.03 \pm 1.86) \cdot 10^{-4}$ & 0.75 \\
\hline 275 & $0.010 \pm 0.001$ & $0.287 \pm 0.032$ & $0.034 \pm 0.008$ & $0.431 \pm 0.136$ & $0.600 \pm 0.138$ & $(9.38 \pm 3.72) \cdot 10^{-5}$ & 0.61 \\
\hline 325 & 0 & $0.315 \pm 0.035$ & $0.016 \pm 0.016$ & $0.302 \pm 0.063$ & $0.729 \pm 0.067$ & $(7.43 \pm 1.78) \cdot 10^{-5}$ & 0.55 \\
\hline 350 & 0 & $0.328 \pm 0.003$ & $0.003 \pm 0.146$ & $0.377 \pm 0.148$ & $0.654 \pm 0.148$ & $(3.53 \pm 1.61) \cdot 10^{-4}$ & 0.52 \\
\hline 375 & 0 & $0.328 \pm 0.082$ & $0.004 \pm 0.004$ & $0.307 \pm 0.183$ & $0.724 \pm 0.184$ & $(4.59 \pm 2.99) \cdot 10^{-5}$ & 0.52 \\
\hline
\end{tabular}

Table S.22 Molalities of aqueous species versus temperature for Solution $10\left[0.855 \mathrm{~mol} \cdot \mathrm{kg}^{-1} \mathrm{UO}_{2} \mathrm{SO}_{4}+2.036 \mathrm{~mol} \cdot \mathrm{kg}^{-1} \mathrm{H}_{2} \mathrm{SO}_{4}\right]$, derived from experimental Raman data

\begin{tabular}{|c|c|c|c|c|c|c|c|}
\hline \multirow[t]{2}{*}{$T /{ }^{\circ} \mathrm{C}$} & \multicolumn{7}{|c|}{ Molality $/ \mathrm{mol} \cdot \mathrm{kg}^{-1}$} \\
\hline & $\mathrm{UO}_{2}{ }^{2+}$ & $\mathrm{UO}_{2} \mathrm{SO}_{4}{ }^{0}$ & $\mathrm{UO}_{2}\left(\mathrm{SO}_{4}\right)_{2}^{2-}$ & $\mathrm{HSO}_{4}^{-}$ & $\mathrm{H}^{+}$ & $\mathrm{SO}_{4}^{2-}$ & $\begin{array}{c}\text { Ionic } \\
\text { Strength }\end{array}$ \\
\hline 25 & $0.191 \pm 0.007$ & $0.314 \pm 0.016$ & $0.350 \pm 0.022$ & $1.421 \pm 0.362$ & $2.650 \pm 0.362$ & $(1.42 \pm 0.42) \cdot 10^{-2}$ & 3.15 \\
\hline 75 & $0.115 \pm 0.004$ & $0.339 \pm 0.018$ & $0.401 \pm 0.026$ & $1.432 \pm 1.927$ & $2.639 \pm 1.927$ & $(4.63 \pm 7.09) \cdot 10^{-3}$ & 3.08 \\
\hline 125 & $0.108 \pm 0.004$ & $0.423 \pm 0.022$ & $0.324 \pm 0.020$ & $1.534 \pm 1.090$ & $2.537 \pm 1.090$ & $(7.48 \pm 6.23) \cdot 10^{-3}$ & 2.91 \\
\hline 175 & $0.139 \pm 0.012$ & $0.506 \pm 0.068$ & $0.209 \pm 0.024$ & $2.019 \pm 0.568$ & $2.053 \pm 0.568$ & $(2.97 \pm 1.19) \cdot 10^{-3}$ & 2.74 \\
\hline 225 & $0.179 \pm 0.016$ & $0.504 \pm 0.078$ & $0.172 \pm 0.035$ & $1.389 \pm 0.940$ & $2.683 \pm 0.940$ & $(4.32 \pm 3.31) \cdot 10^{-4}$ & 2.74 \\
\hline 275 & $0.076 \pm 0.008$ & $0.677 \pm 0.100$ & $0.101 \pm 0.020$ & $1.478 \pm 0.564$ & $2.593 \pm 0.565$ & $(1.80 \pm 0.80) \cdot 10^{-4}$ & 2.39 \\
\hline 325 & 0 & $0.750 \pm 0.081$ & $0.105 \pm 0.018$ & $1.274 \pm 0.222$ & $2.798 \pm 0.222$ & $(4.16 \pm 0.85) \cdot 10^{-4}$ & 2.25 \\
\hline 350 & 0 & $0.745 \pm 0.024$ & $0.110 \pm 0.702$ & $1.262 \pm 0.702$ & $2.809 \pm 0.702$ & $(3.88 \pm 2.38) \cdot 10^{-3}$ & 2.26 \\
\hline 375 & 0 & $0.753 \pm 1.170$ & $0.101 \pm 0.279$ & $1.165 \pm 2.251$ & $2.906 \pm 2.251$ & $(1.29 \pm 2.69) \cdot 10^{-4}$ & 2.24 \\
\hline
\end{tabular}


Table S.23 Molalities of aqueous species versus temperature for Solution $11\left[0.567 \mathrm{~mol} \cdot \mathrm{kg}^{-1} \mathrm{UO}_{2} \mathrm{SO}_{4}+1.349 \mathrm{~mol} \cdot \mathrm{kg}^{-1} \mathrm{H}_{2} \mathrm{SO}_{4}\right]$, derived from experimental Raman data

\begin{tabular}{cccccccc}
\hline$T /{ }^{\circ} \mathrm{C}$ & \multicolumn{7}{c}{ Molality / mol $\cdot \mathrm{kg}^{-1}$} \\
\cline { 2 - 7 } & $\mathrm{UO}_{2}{ }^{2+}$ & $\mathrm{UO}_{2} \mathrm{SO}_{4}{ }^{0}$ & $\mathrm{UO}_{2}\left(\mathrm{SO}_{4}\right)_{2}{ }^{2-}$ & $\mathrm{HSO}_{4}{ }^{-}$ & $\mathrm{H}^{+}$ & $\mathrm{SO}_{4}{ }^{2-}$ & $\mathrm{Strength}^{-}$ \\
\hline 25 & $0.135 \pm 0.006$ & $0.187 \pm 0.012$ & $0.244 \pm 0.017$ & $0.920 \pm 0.343$ & $1.778 \pm 0.349$ & $(1.94 \pm 0.83) \cdot 10^{-2}$ & 2.15 \\
75 & $0.061 \pm 0.003$ & $0.235 \pm 0.015$ & $0.271 \pm 0.020$ & $0.966 \pm 0.898$ & $1.733 \pm 0.900$ & $(6.49 \pm 6.92) \cdot 10^{-3}$ & 2.03 \\
125 & $0.082 \pm 0.004$ & $0.272 \pm 0.017$ & $0.213 \pm 0.016$ & $1.038 \pm 0.604$ & $1.661 \pm 0.608$ & $(6.47 \pm 4.47) \cdot 10^{-3}$ & 1.95 \\
175 & $0.092 \pm 0.005$ & $0.315 \pm 0.021$ & $0.160 \pm 0.013$ & $1.116 \pm 0.429$ & $1.582 \pm 0.434$ & $(1.79 \pm 0.85) \cdot 10^{-3}$ & 1.86 \\
225 & $0.024 \pm 0.002$ & $0.470 \pm 0.041$ & $0.072 \pm 0.014$ & $0.823 \pm 0.288$ & $1.876 \pm 0.296$ & $(2.76 \pm 1.08) \cdot 10^{-4}$ & 1.54 \\
275 & $0.001 \pm 0.004$ & $0.533 \pm 0.040$ & $0.032 \pm 0.006$ & $0.887 \pm 0.360$ & $1.812 \pm 0.365$ & $(1.13 \pm 0.52) \cdot 10^{-4}$ & 1.42 \\
325 & $0.018 \pm 0.002$ & $0.487 \pm 0.069$ & $0.062 \pm 0.014$ & $0.626 \pm 0.171$ & $2.072 \pm 0.183$ & $(1.80 \pm 0.53) \cdot 10^{-4}$ & 1.51 \\
350 & 0 & $0.499 \pm 0.009$ & $0.068 \pm 0.139$ & $0.512 \pm 0.154$ & $2.187 \pm 0.154$ & $(9.84 \pm 2.85) \cdot 10^{-4}$ & 1.49 \\
375 & $0.014 \pm 0.001$ & $0.481 \pm 0.050$ & $0.072 \pm 0.011$ & $0.460 \pm 0.196$ & $2.238 \pm 0.206$ & $(7.03 \pm 3.10) \cdot 10^{-5}$ & 1.52 \\
\hline
\end{tabular}

Table S.24 Molalities of aqueous species versus temperature for Solution $12\left[0.338 \mathrm{~mol} \cdot \mathrm{kg}^{-1} \mathrm{UO}_{2} \mathrm{SO}_{4}+0.805 \mathrm{~mol} \cdot \mathrm{kg}^{-1} \mathrm{H}_{2} \mathrm{SO}_{4}\right]$, derived from experimental Raman data

\begin{tabular}{|c|c|c|c|c|c|c|c|}
\hline \multirow[t]{2}{*}{$T /{ }^{\circ} \mathrm{C}$} & \multicolumn{7}{|c|}{ Molality $/ \mathrm{mol} \cdot \mathrm{kg}^{-1}$} \\
\hline & $\mathrm{UO}_{2}^{2+}$ & $\mathrm{UO}_{2} \mathrm{SO}_{4}{ }^{0}$ & $\mathrm{UO}_{2}\left(\mathrm{SO}_{4}\right)_{2}^{2-}$ & $\mathrm{HSO}_{4}^{-}$ & $\mathrm{H}^{+}$ & $\mathrm{SO}_{4}^{2-}$ & $\begin{array}{c}\text { Ionic } \\
\text { Strength }\end{array}$ \\
\hline 25 & $0.064 \pm 0.003$ & $0.123 \pm 0.008$ & $0.150 \pm 0.011$ & $0.524 \pm 0.242$ & $1.085 \pm 0.245$ & $(2.11 \pm 1.10) \cdot 10^{-2}$ & 1.28 \\
\hline 75 & $0.033 \pm 0.002$ & $0.140 \pm 0.010$ & $0.165 \pm 0.012$ & $0.721 \pm 0.566$ & $0.889 \pm 0.568$ & $(1.11 \pm 1.12) \cdot 10^{-2}$ & 1.22 \\
\hline 125 & $0.056 \pm 0.003$ & $0.157 \pm 0.011$ & $0.124 \pm 0.010$ & $0.918 \pm 0.544$ & $0.692 \pm 0.546$ & $(1.08 \pm 1.07) \cdot 10^{-2}$ & 1.19 \\
\hline 175 & $0.054 \pm 0.004$ & $0.170 \pm 0.014$ & $0.114 \pm 0.011$ & $0.898 \pm 0.403$ & $0.712 \pm 0.405$ & $(2.52 \pm 1.84) \cdot 10^{-3}$ & 1.15 \\
\hline 225 & $0.060 \pm 0.004$ & $0.186 \pm 0.013$ & $0.091 \pm 0.014$ & $0.710 \pm 0.232$ & $0.899 \pm 0.235$ & $(4.18 \pm 1.77) \cdot 10^{-4}$ & 1.11 \\
\hline 275 & $0.031 \pm 0.004$ & $0.266 \pm 0.047$ & $0.041 \pm 0.009$ & $0.520 \pm 0.207$ & $1.089 \pm 0.210$ & $(8.51 \pm 3.80) \cdot 10^{-5}$ & 0.95 \\
\hline 325 & 0 & $0.313 \pm 0.023$ & $0.025 \pm 0.003$ & $0.464 \pm 0.064$ & $1.145 \pm 0.074$ & $(1.25 \pm 0.21) \cdot 10^{-4}$ & 0.85 \\
\hline 350 & 0 & $0.310 \pm 0.003$ & $0.028 \pm 0.117$ & $0.405 \pm 0.123$ & $1.205 \pm 0.123$ & $(5.23 \pm 1.65) \cdot 10^{-4}$ & 0.86 \\
\hline 375 & $0.011 \pm 0.001$ & $0.294 \pm 0.027$ & $0.033 \pm 0.004$ & $0.338 \pm 0.223$ & $1.271 \pm 0.226$ & $(5.78 \pm 3.96) \cdot 10^{-5}$ & 0.89 \\
\hline
\end{tabular}


Table S.25 Fitted parameters for an extended van t'Hoff equation (S.1) to Reactions (1), (2) and (4).**

\begin{tabular}{lcccccc}
\hline & $A / 10^{3}$ & $B / 10^{3}$ & $C$ & $D / 10^{3}$ & $E / 10^{6}$ & -2 \\
\hline Reaction (1) & -259.9 & 4.117 & 0.5294 & -1.461 & 16.76 & 6.863 \\
Reaction (2) & -120.9 & 1.965 & 0.2726 & -0.704 & -2 \\
Reaction (4) & -107.9 & 1.91 & 0.255 & -0.683 & 6.83 & 1 \\
\hline
\end{tabular}

* The following extended van't Hoff Equation was fitted to the temperature dependent formation constants for Reactions (1) and (2) derived in the manuscript, as well as those of Reaction (4) of Marshall and Jones (Ref 37),

$$
\log K=\frac{A}{T}+B+C T+D \log T+\frac{E}{T^{2}}+W \log K_{w}
$$

where $K_{\mathrm{w}}$ is the temperature-dependent ionization constant of water from Marshall and Franck (Ref. S.1).

** Reaction (1): $\mathrm{UO}_{2}{ }^{2+}(\mathrm{aq})+\mathrm{SO}_{4}{ }^{2-}(\mathrm{aq}) \rightleftharpoons \mathrm{UO}_{2} \mathrm{SO}_{4}{ }^{0}(\mathrm{aq})$

$* *$ Reaction (2): $\mathrm{UO}_{2}{ }^{2+}(\mathrm{aq})+2 \mathrm{SO}_{4}{ }^{2-}(\mathrm{aq}) \rightleftharpoons \mathrm{UO}_{2}\left(\mathrm{SO}_{4}\right)_{2}{ }^{2-}(\mathrm{aq})$

**Reaction (4): $\mathrm{HSO}_{4}^{-}(\mathrm{aq}) \rightleftharpoons \mathrm{H}^{+}(\mathrm{aq})+\mathrm{SO}_{4}{ }^{2-}(\mathrm{aq})$ 
Table S.26 Interaction coefficients $\varepsilon(j, k)$ in $\mathrm{mol}^{-1} \cdot \mathrm{kg}$ in the PHREEQC SIT database version 9b0*, used in Equation (13)

\begin{tabular}{cccc}
\hline Species $j$; Species $k \rightarrow$ & $\mathrm{Cl}^{-}(\mathrm{aq})$ & $\mathrm{ClO}_{4}^{-}(\mathrm{aq})$ & $\mathrm{NO}_{3}^{-}(\mathrm{aq})$ \\
& & & \\
\hline $\mathrm{H}^{+}(\mathrm{aq})$ & $0.12 \pm 0.01$ & $0.14 \pm 0.02$ & $0.07 \pm 0.01$ \\
$\mathrm{UO}_{2}{ }^{2+}(\mathrm{aq})$ & $0.21 \pm 0.02$ & $0.46 \pm 0.03$ & $0.24 \pm 0.03$ \\
\hline Species $j$; Species $k \rightarrow$ & $\mathrm{Li}^{+}(\mathrm{aq})$ & $\mathrm{Na}^{+}(\mathrm{aq})$ & $\mathrm{K}^{+}(\mathrm{aq})$ \\
\hline $\mathrm{OH}^{-}(\mathrm{aq})$ & $-0.02 \pm 0.03$ & $0.04 \pm 0.01$ & $0.09 \pm 0.01$ \\
$\mathrm{HSO}_{4}^{-}(\mathrm{aq})$ & $\mathrm{NA}$ & $-0.01 \pm 0.02$ & $-0.06 \pm 0.02$ \\
$\mathrm{SO}_{4}{ }^{-2}(\mathrm{aq})$ & $-0.03 \pm 0.04$ & $-0.12 \pm 0.60$ & $-0.12 \pm 0.06$ \\
$\mathrm{UO}_{2}\left(\mathrm{SO}_{4}\right)_{2}{ }^{2-}(\mathrm{aq})$ & $\mathrm{NA}$ & &
\end{tabular}

*Refs. S.2-4 


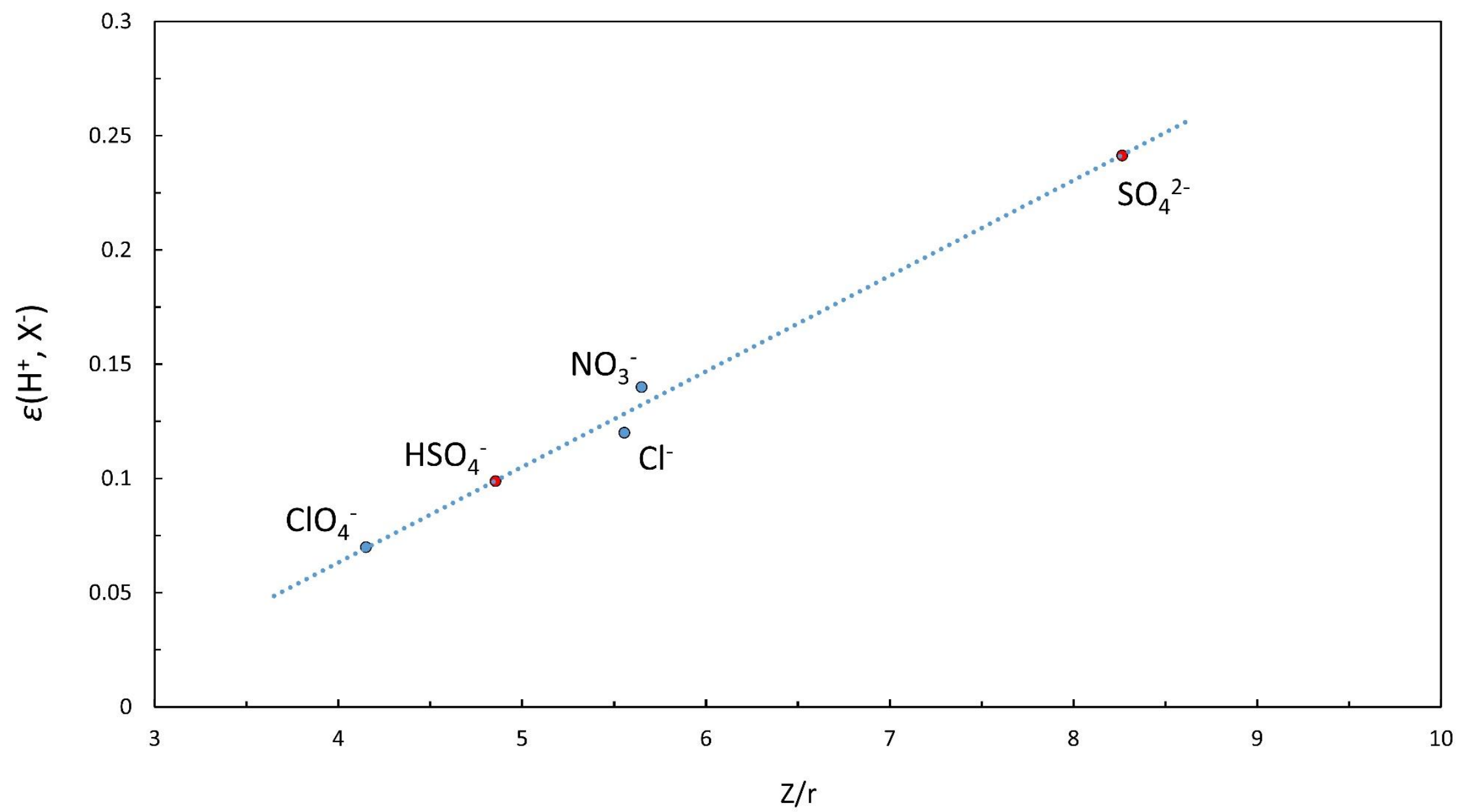

Fig S.1: Linear Fit of the Interaction Parameters $\varepsilon\left(\mathrm{H}^{+}, \mathrm{X}^{-}\right)\left(\mathrm{X}^{-}=\mathrm{ClO}_{4}{ }^{-}, \mathrm{NO}_{3}{ }^{-}, \mathrm{Cl}^{-}\right)$versus $\mathrm{Z} / r$, for the estimation of $\varepsilon\left(\mathrm{H}^{+}, \mathrm{SO}_{4}{ }^{2-}\right)$ and $\varepsilon\left(\mathrm{H}^{+}, \mathrm{HSO}_{4}{ }^{-}\right)$ 


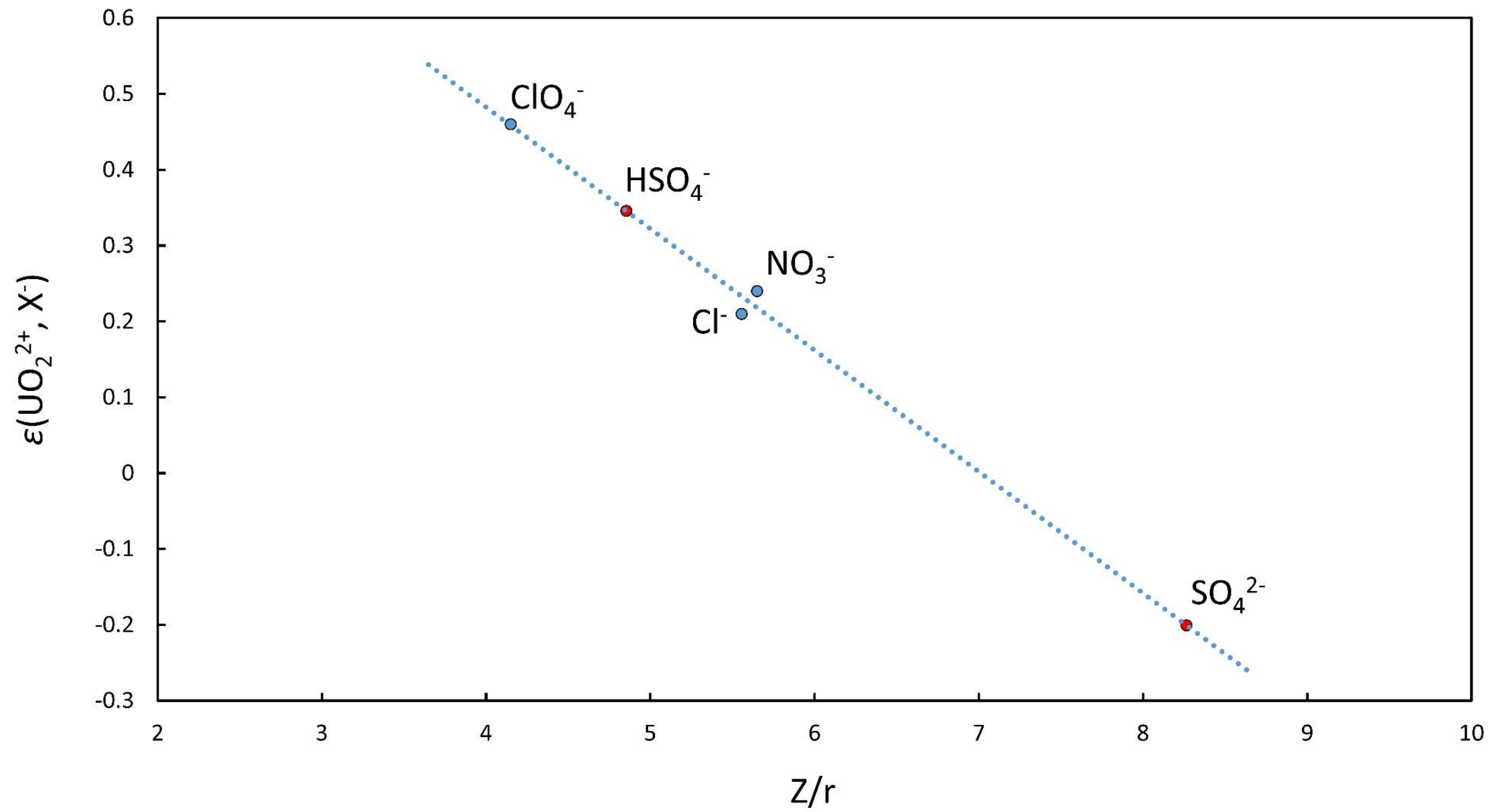

Fig S.2: Linear Fit of the Interaction Parameters $\varepsilon\left(\mathrm{UO}_{2}{ }^{2+}, \mathrm{X}^{-}\right)\left(\mathrm{X}^{-}=\mathrm{ClO}_{4}^{-}, \mathrm{NO}_{3}^{-}, \mathrm{Cl}^{-}\right)$versus $Z / r$, for the estimation of $\varepsilon\left(\mathrm{UO}_{2}{ }^{2+}, \mathrm{SO}_{4}{ }^{2-}\right)$ and $\varepsilon\left(\mathrm{UO}_{2}^{2+}, \mathrm{HSO}_{4}^{-}\right)$ 


\section{References}

1. Marshall, W. L.; Franck, E., Ion Product of Water Substance, 0-1000 ${ }^{\circ} \mathrm{C}, 1-10,000$ Bars New International Formulation and its Background. J. Phys. Chem. Ref. Data 1981, 10, 295-304.

2. Giffaut, E.; Grivé, M.; Blanc, P.; Vieillard, P.; Colàs, E.; Gailhanou, H.; Gaboreau, S.; Marty, N.; Madé, B.; Duro, L., Andra Thermodynamic Database for Performance Assessment: Thermochimie. Appl. Geochem. 2014, 49, 225-236.

3. Guillaumont, R.; Fanghanel, T.; Fuger, J.; Grenthe, I.; Neck, V.; Palmer, D. A.; Rand, M. A., Update on the Chemical Thermodynamics of Uranium, Neptunium, Plutonium, Americium and Technetium. Elsevier B.V.: Amsterdam, 2003.

4. Tian, G.; Rao, L., Spectrophotometric and Calorimetric Studies of U(VI) Complexation with Sulfate at (25 to 70) degrees C. J. Chem. Thermodyn. 2009, 41, 569-574. 